\title{
A New Two-Dimensional Map with Hidden Attractors
}

\author{
Chuanfu Wang (1) and Qun Ding * \\ Electronic Engineering College, Heilongjiang University, Harbin 150080, China; 1172054@s.hlju.edu.cn \\ * Correspondence: 1984008@hlju.edu.cn
}

Received: 31 January 2018; Accepted: 24 April 2018; Published: 27 April 2018

\begin{abstract}
The investigations of hidden attractors are mainly in continuous-time dynamic systems, and there are a few investigations of hidden attractors in discrete-time dynamic systems. The classical chaotic attractors of the Logistic map, Tent map, Henon map, Arnold's cat map, and other widely-known chaotic attractors are those excited from unstable fixed points. In this paper, the hidden dynamics of a new two-dimensional map inspired by Arnold's cat map is investigated, and the existence of fixed points and their stabilities are studied in detail.
\end{abstract}

Keywords: hidden attractors; fixed point; stability

\section{Introduction}

The investigations of the chaotic system were greatly encouraged by the discovery of the Lorenz system [1]. The Lorenz system is one of the most wildly-studied continuous-time dynamic systems, and other classical continuous-time dynamic systems include the Rössler system, Chua system, Chen system, Lü system, and Sprott system [2-6]. Most attractors of those classical continuous-time dynamic systems are excited from unstable equilibria. However, hidden attractors imply the basin of attraction does not contain neighborhoods of equilibria [7]. For finding hidden attractors, a lot of systems improved when classical continuous-time dynamic systems were proposed [8-19]. These investigations of hidden attractors can be classified by the number and stability of equilibria, such as no equilibrium, finite stable equilibria, and infinite stable equilibria. In 2010, a special analytical-numerical algorithm of finding hidden attractors in Chua system was proposed [20]. The special algorithm can find the accuracy initial values that lead to hidden attractors and has promoted the development of finding hidden attractors in continuous-time dynamic systems [21-24]. However, most investigations of hidden attractors are mainly in continuous-time dynamic systems, such as the Chen system, Sprott system, Chua system, and Lü system [15-24]. There are only a few of investigations of hidden attractors in discrete-time systems.

The classical chaotic maps include the Logistic map, Tent map, Henon map, and Arnold's cat map [25-28]. In line with continuous-time dynamic systems, these hidden attractors in the classical chaotic maps are also excited from unstable fixed points. In 2016, Jafari et al. studied a new one-dimensional chaotic map with no fixed point inspired by Logistic map, and its bifurcation and period doubling were introduced [29]. At the same year, Jiang et al. performed a search to find hidden attractors in a new two-dimensional chaotic map inspired by the Henon map and analyzed several different cases on fixed points, such as no fixed point, single fixed point, and two fixed points [30].

In this paper, a new two-dimensional chaotic map inspired by Arnold's cat map is proposed. For the limitation in the form of the Arnold's cat map, the number of fixed points in the new two-dimensional chaotic map is not larger than two, and the fixed points are closely related to the Lyapunov exponents. Due to the restriction of the form of the Arnold's cat map, the new two-dimensional chaotic attractor can only appear in the case of no fixed point. Thus, our concern 
focuses on the case of no fixed point. The paper is arranged as follows: Section 2 describes the Arnold's cat map and shows its chaotic attractor. Section 3 analyzes the stability of the equilibria in the new two-dimensional map. Section 4 demonstrates the digitalization and hardware implementation of the new two-dimensional map. The complexity of output time series is tested by approximate entropy in Section 5, and Section 6 summarizes the conclusions of this paper.

\section{Arnold's Cat Map}

Arnold's cat map, also known as cat chaotic map, is a chaotic map of repeated folding and stretching in a limited area and is wildly used in multimedia chaotic encryption [28]. Arnold's cat map is a two-dimensional chaotic map and defined as

$$
\left[\begin{array}{l}
x(n+1) \\
y(n+1)
\end{array}\right]=\left[\begin{array}{ll}
1 & 1 \\
1 & 2
\end{array}\right]\left[\begin{array}{l}
x(n) \\
y(n)
\end{array}\right](\bmod 1)
$$

Equation (1) can be transformed into Equation (2) for calculating the fixed points.

$$
\left\{\begin{array}{l}
x(n)=x(n)+y(n)(\bmod 1) \\
y(n)=x(n)+2 y(n)(\bmod 1)
\end{array}\right.
$$

Equation (2) always has a fixed point, because it is composed of homogeneous linear equations. The fixed point of the Arnold's cat map is $\left\{\begin{array}{l}x^{*}=0 \\ y^{*}=0\end{array}\right.$. The Jacobian matrix of Arnold's cat map is $\left[\begin{array}{ll}1 & 1 \\ 1 & 2\end{array}\right]$, and the two eigenvalues are calculated as $\operatorname{Eig}_{1}=\frac{3+\sqrt{5}}{2} \approx 2.618$ and $\operatorname{Eig}_{2}=\frac{3-\sqrt{5}}{2} \approx$ 0.382 . Therefore, the fixed point $(0,0)$ is an unstable fixed point. It is a saddle point, because there is a positive Lyapunov exponent and a negative Lyapunov exponent. For a positive Lyapunov exponent, Arnold's cat map is a two-dimensional chaotic map. In Arnold's cat map, two eigenvalues of the Jacobian matrix are associated, respectively, to an expanding and a contracting eigenspace, which are also the stable and unstable manifolds [31]. The phase diagram of Arnold's cat map is shown in Figure 1.

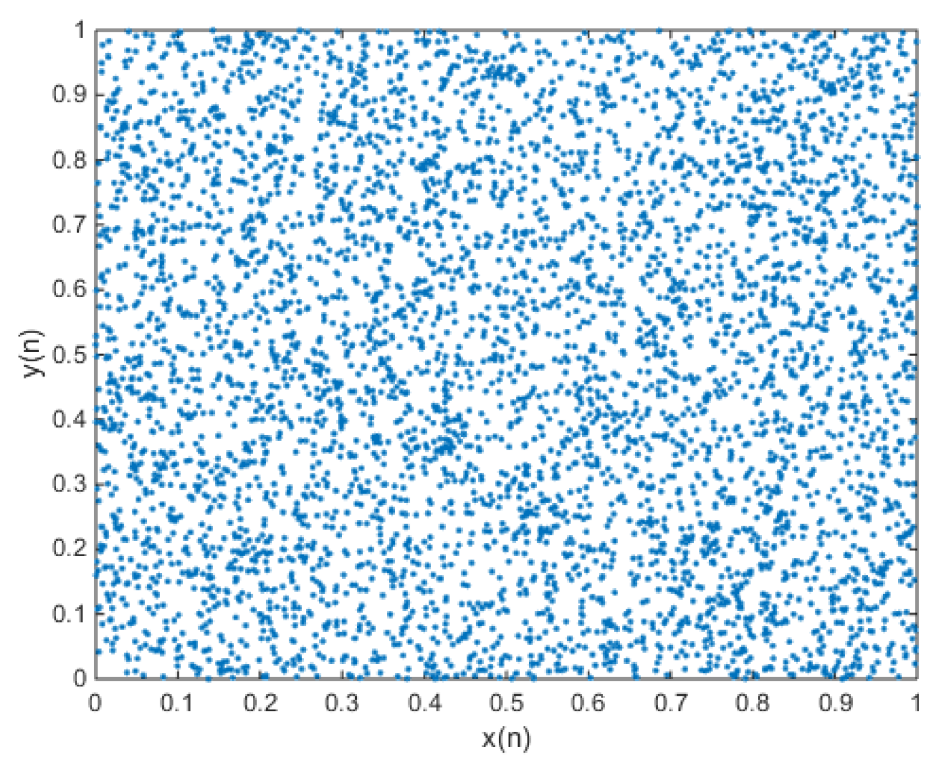

Figure 1. When $x(0)=0.7$ and $y(0)=0.6$, this represents the phase diagram of Arnold's cat map. 
The generalized Arnold's cat map is defined as

$$
\left[\begin{array}{l}
x(n+1) \\
y(n+1)
\end{array}\right]=\left[\begin{array}{cc}
1 & a \\
b & a b+1
\end{array}\right]\left[\begin{array}{l}
x(n) \\
y(n)
\end{array}\right](\bmod 1) .
$$

The fixed point of the generalized Arnold's cat map also is $\left\{\begin{array}{l}x^{*}=0 \\ y^{*}=0\end{array}\right.$. The Jacobian matrix of the generalized Arnold's cat map is, and two eigenvalues are $\lambda_{1}=1+\frac{a b+\sqrt{(a b+2)^{2}-4}}{2}>1$ and $\lambda_{2}=1+\frac{a b-\sqrt{(a b+2)^{2}-4}}{2}<1$. Therefore, the fixed point $(0,0)$ also is an addle point. The generalized Arnold's cat map also is chaotic map, because it has one positive Lyapunov exponent. Despite Arnold's cat map or the generalized Arnold's cat map, they always have unstable saddle pint $(0,0)$.

\section{A New Two-Dimensional Chaotic Map without Fixed Points}

In this paper, a new two-dimensional chaotic map inspired by Arnold's cat map is proposed. It is defined as

$$
\left[\begin{array}{l}
x(n+1) \\
y(n+1)
\end{array}\right]=\left[\begin{array}{ll}
a & b \\
c & d
\end{array}\right]\left[\begin{array}{l}
x(n) \\
y(n)
\end{array}\right]+\left[\begin{array}{l}
e \\
f
\end{array}\right](\bmod 1) .
$$

in which $a=k c+1, b=k(d-1), e \neq k f \neq 0, e \in(0,1), f \in(0,1)$, and $k \neq 0$. For calculating the fixed point, Equation (4) can be transformed into two-dimensional equations.

$$
\left\{\begin{array}{l}
x(n)=(k c+1) x(n)+k(d-1) y(n)+e(\bmod 1) \\
y(n)=c x(n)+d y(n)+f(\bmod 1)
\end{array}\right.
$$

Equation (5) can be transformed into nonhomogeneous linear equations by collecting the like terms.

$$
\left\{\begin{array}{l}
k c x(n)+k(d-1) y(n)=-e(\bmod 1) \\
c x(n)+(d-1) y(n)=-f(\bmod 1)
\end{array}\right.
$$

There is no solution to the nonhomogeneous linear equations. Thus, the map (4) has no fixed point. However, the coefficients should be further limited for obtaining hidden chaotic attractors. The Jacobian matrix of the map (4) is

$$
J_{1}=\left[\begin{array}{cc}
k c+1 & k d-k \\
c & d
\end{array}\right]
$$

The characteristic equation of the matrix $J_{1}$ is calculated as

$$
\operatorname{det}\left(\lambda I-J_{1}\right)=\lambda^{2}-\operatorname{tr}\left(J_{1}\right) \lambda+\operatorname{det}\left(J_{1}\right)=0 .
$$

in which $\operatorname{det}\left(J_{1}\right)=d+c k$ is the determinant of matrix $J_{1}$ and $\operatorname{tr}\left(J_{1}\right)=k c+1+d$ is the trace of matrix $J_{1}$. The characteristic equation of the matrix $J_{1}$ is a quadratic function. The roots of the Equation (8) are

$$
\lambda_{1,2}=\frac{k c+1+d \pm(k c+d-1)}{2}
$$

in which $\lambda_{1}=1$ and $\lambda_{2}=k c+d$. The two corresponding Lyapunov exponents are $L E_{1}=\ln \left|\lambda_{1}\right|=0$ and $L E_{2}=\ln |k c+d|$. The non-chaos fixed point attractors have negative Lyapunov exponents. The non-chaos periodic or limit cycle attractors have non-positive Lyapunov exponents. The chaotic attractors have positive Lyapunov exponents. Therefore, the chaotic system exists at least a positive Lyapunov exponent. For obtaining hidden chaotic attractors, the second eigenvalue $\lambda_{2}=k c+d$ 
should be larger than 1 . For example, the coefficients are set as $c=1, d=2$, and $k=2$, and $|k c+d|=4>1$. Combining with the map (4), the new two-dimensional map with no fixed point is defined as

$$
\left[\begin{array}{l}
x(n+1) \\
y(n+1)
\end{array}\right]=\left[\begin{array}{ll}
3 & 2 \\
1 & 2
\end{array}\right]\left[\begin{array}{l}
x(n) \\
y(n)
\end{array}\right]+\left[\begin{array}{l}
0.1 \\
0.2
\end{array}\right](\bmod 1)
$$

in which $e=0.1$ and $f=0.2$. The phase diagram of attractors is shown in Figure 2, and the plot of the output time series is shown in Figure 3.

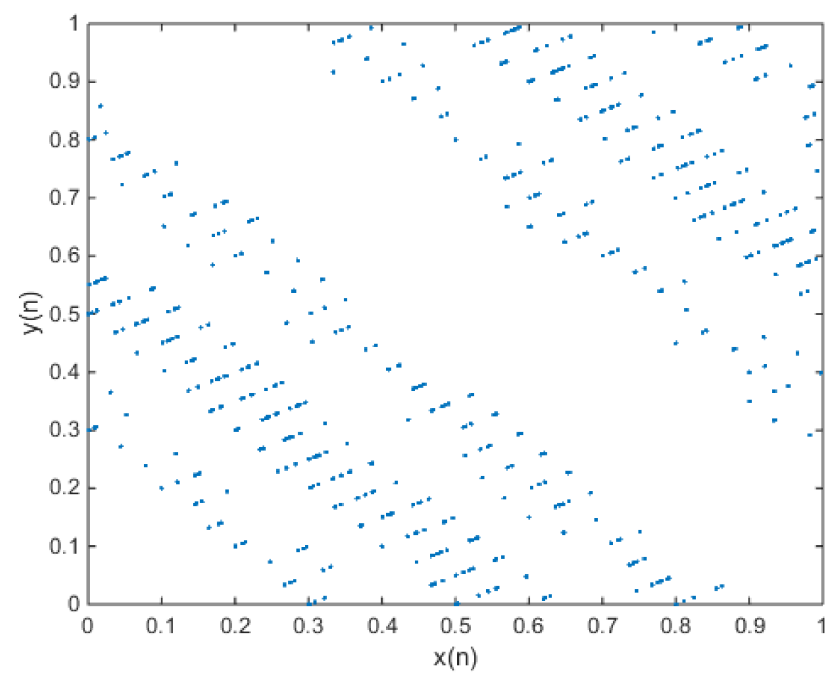

Figure 2. When $x(0)=0.7$ and $y(0)=0.6$, the phase diagram of the new 2-D map with $a=3$, $b=2, c=1, d=2, e=0.1$, and $f=0.2$.

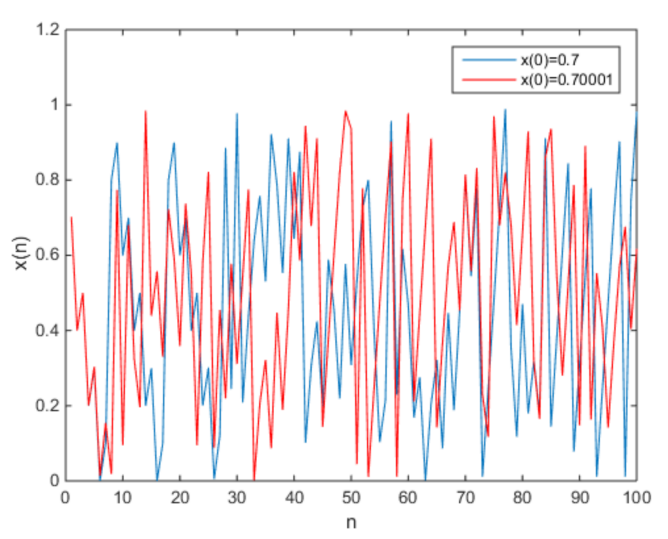

(a)

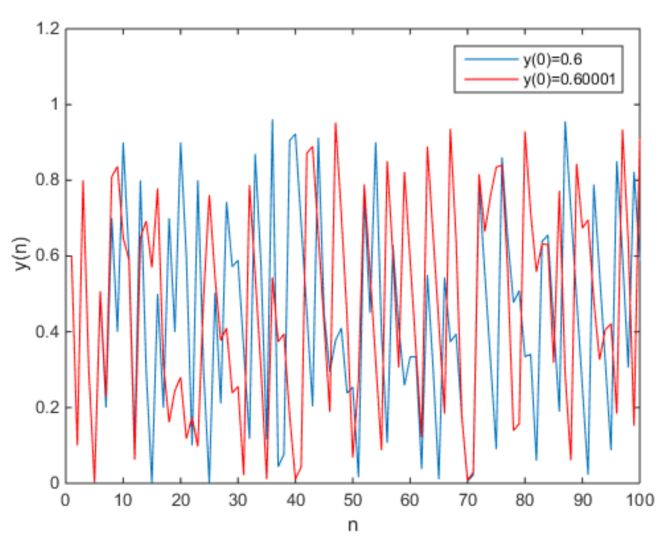

(b)

Figure 3. The plot of the output time series (a) $x(n),(\mathbf{b}) y(n)$.

The new two-dimensional map (10) is a chaotic map, because it has a positive Lyapunov exponent.

From Equation (9), the chaotic behavior in the map (4) is dependent only on the coefficients $c, d$, and $k$, and the second eigenvalue of the Jacobian matrix $J_{1}$ is the simple combination of $c, d$, and $k$. The Lyapunov exponent can be changed with different parameters $c, d$, and $k$. When $c=1.1, d=2$, $k=2$, and $t=4.2>1$. Combining with the map (4), a new two-dimensional map without fixed points is defined as 


$$
\left[\begin{array}{l}
x(n+1) \\
y(n+1)
\end{array}\right]=\left[\begin{array}{ll}
3.2 & 2 \\
1.1 & 2
\end{array}\right]\left[\begin{array}{l}
x(n) \\
y(n)
\end{array}\right]+\left[\begin{array}{l}
0.1 \\
0.2
\end{array}\right](\bmod 1) .
$$

in which $e=0.1$ and $f=0.2$. The phase diagram of the chaotic attractors is shown in Figure 4 , and the plot of the output time series is shown in Figure 5.

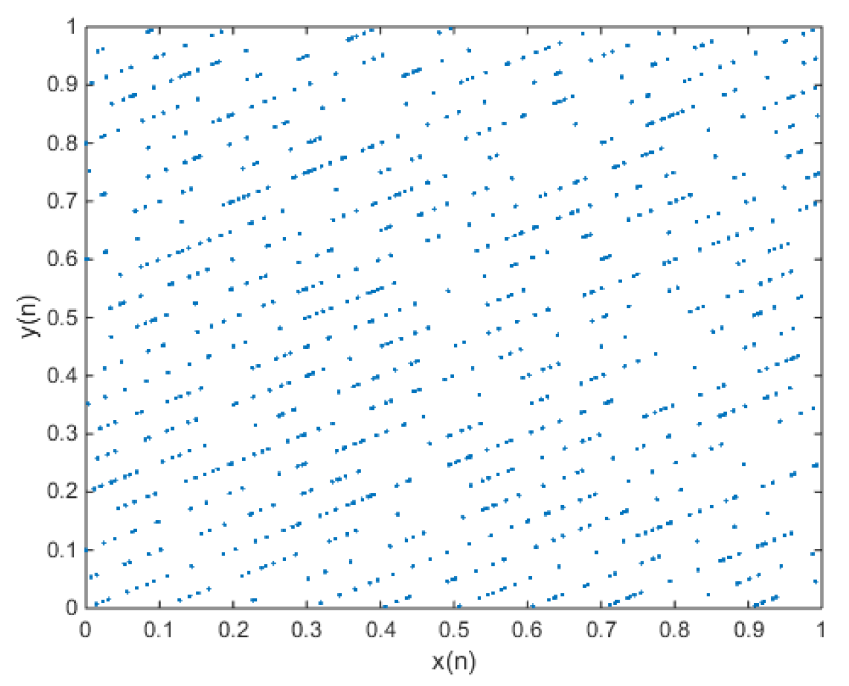

Figure 4. When $x(0)=0.7$ and $y(0)=0.6$, the phase diagram of the new 2-D map with $a=3.2$, $b=2, c=1.1, d=2, e=0.1$, and $f=0.2$.

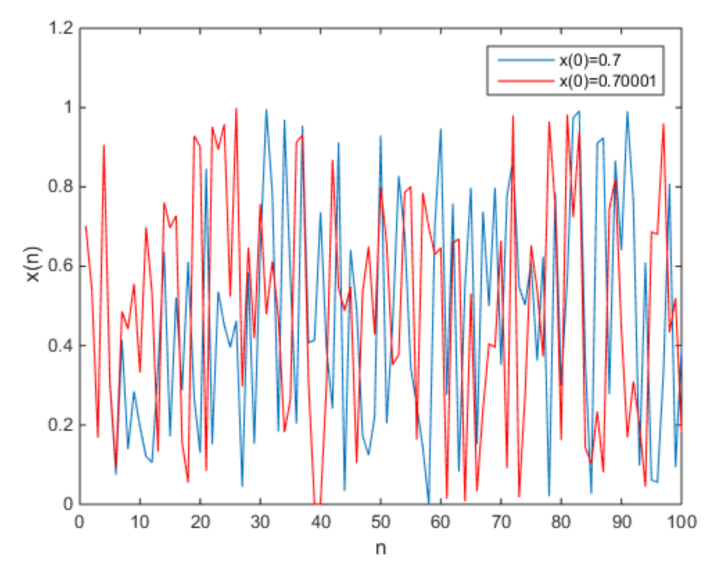

(a)

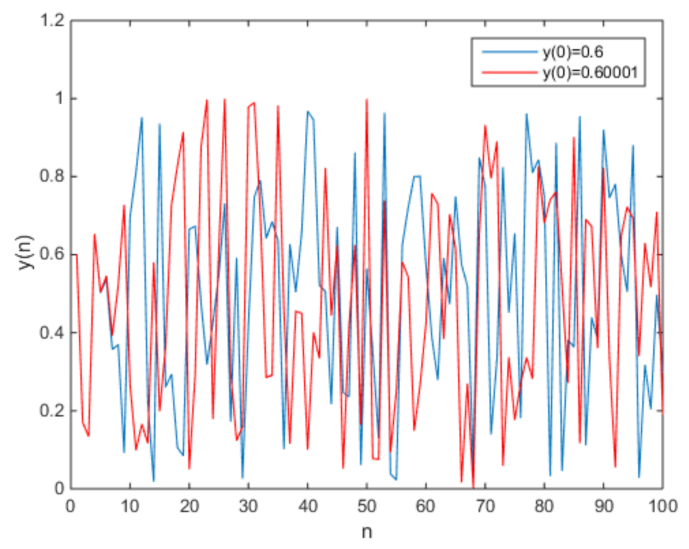

(b)

Figure 5. The plot of the output time series (a) $x(n),(\mathbf{b}) y(n)$.

As can be observed from the plots of the time series, it is obvious that the map (11) has initial value sensitivity, randomness, and so on. For no fixed point in the map (11), the map (11) is a chaotic map and has a hidden chaotic attractor. As can be seen from Figure 1, the chaotic attractor of the map (11) is not similar to that of Arnold's cat map. When $c=-0.25, d=2, k=2$, and $t=1.5>1$. Combining with the map (4), a new two-dimensional map is defined as

$$
\left[\begin{array}{l}
x(n+1) \\
y(n+1)
\end{array}\right]=\left[\begin{array}{cc}
0.5 & 2 \\
-0.25 & 2
\end{array}\right]\left[\begin{array}{l}
x(n) \\
y(n)
\end{array}\right]+\left[\begin{array}{l}
0.1 \\
0.2
\end{array}\right](\bmod 1) .
$$

in which $e=0.1$ and $f=0.2$. The phase diagram of the chaotic attractors is shown in Figure 6, and the plot of the output time series is shown in Figure 7. 


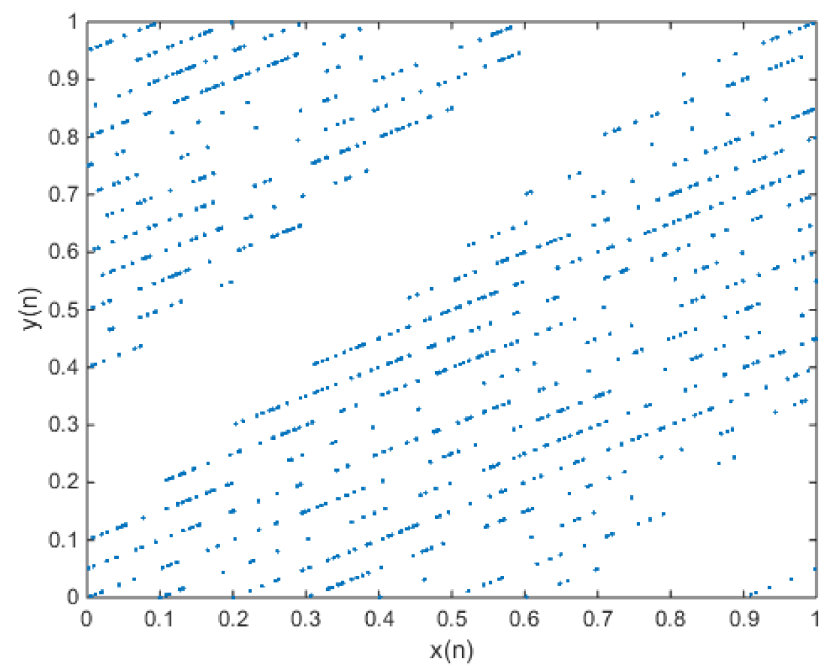

Figure 6. When $x(0)=0.7$ and $y(0)=0.6$, the phase diagram of the new 2-D map with $a=0.5$, $b=2, c=-0.25, d=2, e=0.1$, and $f=0.2$.

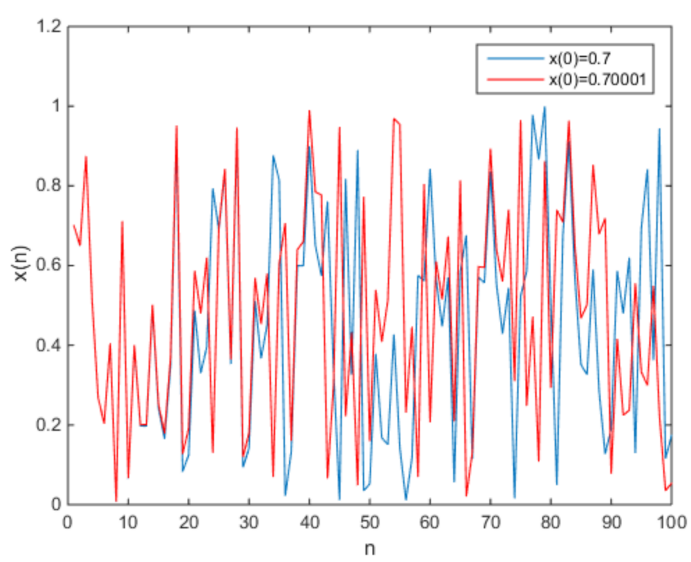

(a)

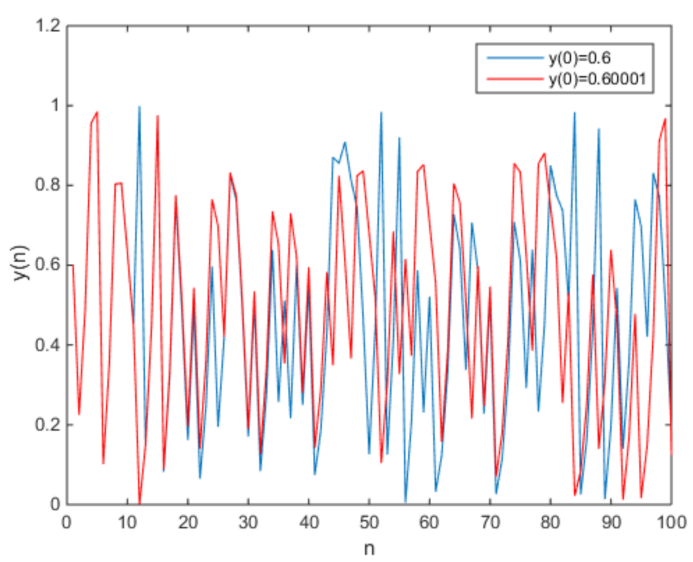

(b)

Figure 7. The plot of the output time series (a) $x(n),(\mathbf{b}) y(n)$.

Compared with Figure 1, the phase diagram of the map (12) is dissimilar to the Figures 2 and 4. When initial value is changed, $x(n)$ has the same output time series in the first 20 iterations, and $y(n)$ has the same output time series in the first 10 iterations.

\section{Digitalization and Hardware Implementation}

For the digitalization of information, chaotic systems need to be digitized before they are used [32,33]. The new two-dimensional chaotic map can be digitalized in two ways: one is the floating-point representation, the other is fixed-point representation. According to IEEE 754-2008 [34], floating-point is divided into single precision and double precision. The form of floating-point is shown in Table 1.

Table 1. Floating-point representation.

\begin{tabular}{cccc}
\hline Floating-Point & Sign & Exponent & Fraction \\
\hline Single precision & $1 \mathrm{bit}$ & $8 \mathrm{bits}$ & $23 \mathrm{bits}$ \\
Double precision & $1 \mathrm{bit}$ & $11 \mathrm{bits}$ & $52 \mathrm{bits}$ \\
\hline
\end{tabular}


The hardware consumptions of the fixed-point representation are not very high, because it includes sign bit and fraction bits. However, there is no standard form in fixed-point representation. Compared with floating-point computing, fixed-point computing is faster, and hardware implementation is smaller [35]. For a better performance in hardware implementation, the new two-dimensional map is represented by fixed-point in this paper. Since $x(n)$ and $y(n)$ are decimal numbers, they are more easily represented by fixed-point. Therefore, $x(n)$ and $y(n)$ are changed from decimals to integers. The digitized $N$ bits of decimal $\beta$ can be represented as

$$
\widetilde{\beta}=\left\lfloor 2^{N} \beta\right\rfloor 2^{-N} .
$$

in which $\widetilde{\beta}$ is an $N$ bits approximation of $\beta$. The digitized $N$ bits of map $\varphi(\beta)=\lambda \beta \bmod 1$ can be written as $\widetilde{\varphi}(\widetilde{\beta})=\left\lfloor 2^{N} \lambda \widetilde{\beta} \bmod 2^{N}\right\rfloor 2^{-N}$, and the digitized $N$ bits of map (4) can be written as

$$
\left\{\begin{array}{l}
\widetilde{x}(n+1)=\left\lfloor 2^{N} a \widetilde{x}(n)+2^{N} b \widetilde{y}(n)+2^{N} e \bmod 2^{N}\right\rfloor 2^{-N} \\
\widetilde{y}(n+1)=\left\lfloor 2^{N} c \widetilde{x}(n)+2^{N} d \widetilde{y}(n)+2^{N} f \bmod 2^{N}\right\rfloor 2^{-N}
\end{array} .\right.
$$

Multiplying both sides by $2^{N}$, map (14) can be represented as

$$
\left\{\begin{array}{l}
2^{N} \widetilde{x}(n+1)=\left\lfloor 2^{N} a \widetilde{x}(n)+2^{N} b \widetilde{y}(n)+2^{N} e \bmod 2^{N}\right\rfloor \\
2^{N} \widetilde{y}(n+1)=\left\lfloor 2^{N} c \widetilde{x}(n)+2^{N} d \widetilde{y}(n)+2^{N} f \bmod 2^{N}\right\rfloor
\end{array} .\right.
$$

Denote $2^{N} \widetilde{x}(n), 2^{N} \widetilde{y}(n), 2^{N} e$, and $2^{N} f$ by $x(n), y(n), e$, and $f$, respectively. The digitized $N$ bits of map (15) can be written as

$$
\left[\begin{array}{l}
x(n+1) \\
y(n+1)
\end{array}\right]=\left\lfloor\left[\begin{array}{ll}
a & b \\
c & d
\end{array}\right]\left[\begin{array}{l}
x(n) \\
y(n)
\end{array}\right]+\left[\begin{array}{l}
e \\
f
\end{array}\right]\right\rfloor\left(\bmod 2^{N}\right),
$$

in which $x(n)$ and $y(n)$ is in the interval $\left[0,2^{N}-1\right], N$ presents the length of finite precision, $x(n)$ and $y(n)$ is represented by $N$ bits. When $N=32$, the hardware implementation by FPGA is shown in Figure 8.

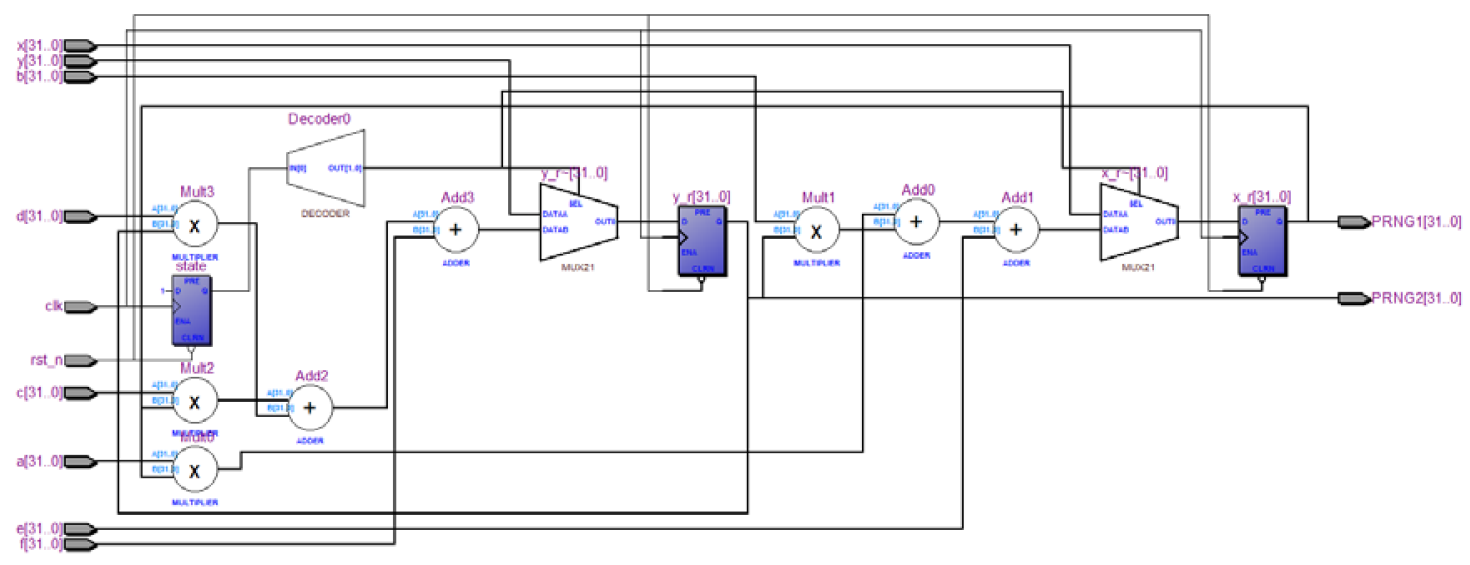

Figure 8. The block diagram of the hardware implementation by FPGA.

\section{The Analysis of Complexity}

The approximate entropy algorithm is proposed from the angle of measuring the complexity of time series [36-39]. The main idea of the approximate entropy algorithm is using a non-negative value to quantify the complexity and irregularity of the time series, and the value increases with the increase of sequence complexity. The calculation process of the approximate entropy is shown as follows: 
1 Suppose the initial data is the sequence $x(1), x(2), \ldots x(N)$, and then divide them into m-dimensional vectors

$$
X(i)=[x(i), x(i+1), \ldots, x(i+m-1)],
$$

in which $i=1,2,3 \ldots N-m+1$.

2 The distance between $x(i)$ and $x(j)$ is defined as

$$
d(i, j)=\max _{k=1-m-1}[|x(i+k)-x(j+k)|] .
$$

3 Setting a threshold value $r(r>0)$, for each $i$, we can obtain the statistics of $d(i, j)$.

$$
C_{i}^{m}(r)=\frac{1}{N-m+1} \operatorname{Sum}\{d(i, j)<r\}
$$

4 The mean of logarithm of $C_{i}{ }^{m}(r)$ is written as $\phi^{m}(r)$ and can be calculated by

$$
\phi^{m}(r)=\frac{1}{N-m+1} \sum_{i=1}^{N-m+1} \ln C_{i}^{m}(r)
$$

5 Changing dimension and repeating step 1 to step 4, we can obtain the approximate entropy

$$
\operatorname{ApEn}(m, r)=\lim _{N \rightarrow \infty}\left[\phi^{m}(r)-\phi^{m+1}(r)\right]
$$

However, in practical terms, the length of the data sequence is bounded. Therefore, the approximate entropy algorithm is changed into

$$
\left.\operatorname{ApEn}(m, r, N)=\phi^{m}(r)-\phi^{m+1}(r)\right]
$$

Pincus found that there exists a minimal dependency between $A p E n$ and $N$ when $m=2$ and $r \in[0.1 S D(x), 0.2 S D(x)]$ [36]. $S D(x)$ is the standard deviation of $x$. The complexity of the output time series of Arnold's cat map and new two-dimensional chaotic maps are tested by approximate entropy algorithm, and the consequence show that the output time series of Equation (11) has a higher complexity. The specific results are shown in Table 2.

Table 2. Approximate entropy test.

\begin{tabular}{cccccc}
\hline Chaotic Map & Time Series & $\boldsymbol{m}$ & $\boldsymbol{r}=\mathbf{0 . 1 5 S D}$ & $\boldsymbol{N}$ & ApEn \\
\hline \multirow{2}{*}{ Equation (1) } & $x(n)$ & 2 & 0.0435 & 2000 & 0.9787 \\
& $y(n)$ & 2 & 0.0436 & 2000 & 0.9963 \\
\hline \multirow{2}{*}{ Equation (10) } & $x(n)$ & 2 & 0.0426 & 2000 & 0.7591 \\
& $y(n)$ & 2 & 0.0437 & 2000 & 0.8841 \\
\hline \multirow{2}{*}{ Equation (11) } & $x(n)$ & 2 & 0.0436 & 2000 & 1.4171 \\
& $y(n)$ & 2 & 0.0434 & 2000 & 1.2831 \\
\hline \multirow{2}{*}{ Equation (12) } & $x(n)$ & 2 & 0.0438 & 2000 & 0.5433 \\
& $y(n)$ & 2 & 0.0428 & 2000 & 0.8429 \\
\hline
\end{tabular}

\section{Conclusions}

In this paper, the hidden attractors for the two-dimensional chaotic map are studied, and the existence of fixed points and their stability are considered. Due to the restriction of the form of the Arnold's cat map, the new two-dimensional chaotic attractor can only appear in the case of no fixed point. The selection of coefficients directly affects whether the two-dimensional map has 
chaotic behavior, because the eigenvalue of the Jacobian matrix of the new two-dimensional chaotic map is a simple combination of the coefficients. Three concrete examples are given to illustrate the relationship between the coefficients and the chaotic behavior. The different coefficients can not only determine the chaotic behavior of the new two-dimensional chaotic map but also affect the shape of the chaotic attractor.

Author Contributions: C.W. conceived and wrote the paper. Q.D. gave some theoretical guidance. All authors have read and approved the final manuscript.

Acknowledgments: This work is supported financially by Natural Science Foundation of China (No. 61471158) and the Innovative Team of the Heilongjiang Province (No. 2012TD007).

Conflicts of Interest: The authors declare no conflict of interest.

\section{References}

1. Lorenz, E.N. Deterministic non-periodic flow. J. Atmos. Sci. 1963, 20, 130-141. [CrossRef]

2. Rössler, O.E. An equation for continuous chaos. Phys. Lett. A 1976, 57, 397-398. [CrossRef]

3. Chua, L.O.; Lin, G.N. Canonical realization of Chua's circuit family. IEEE Trans. Circuits Syst. 1990, 37, 885-902. [CrossRef]

4. Chen, G.; Ueta, T. Yet another chaotic attractor. Int. J. Bifurc. Chaos 1999, 9, 1465-1466. [CrossRef]

5. Lü, J.; Chen, G. A new chaotic attractor coined. Int. J. Bifurc. Chaos 2000, 3, 659-661. [CrossRef]

6. Jafari, S.; Sprott, J.C.; Molaie, M. A simple chaotic flow with a plane of equilibria. Int. J. Bifurc. Chaos 2016, 26, 1650098. [CrossRef]

7. Dawid, D.; Sajad, J.; Tomasz, K.; Nikolay, V.K.; Gennady, A.L.; Awadhesh, P. Hidden attractors in dynamical systems. Phys. Rep. 2016, 637, 1-50.

8. Lai, Q.; Guan, Z.H.; Wu, Y.; Liu, F.; Zhang, D. Generation of multi-wing chaotic attractors from a Lorenz-like system. Int. J. Bifurc. Chaos 2013, 23, 1350152. [CrossRef]

9. Wang, X.; Chen, G. Constructing a chaotic system with any number of equilibria. Nonlinear Dyn. 2013, 71, 429-436. [CrossRef]

10. Jafari, S.; Sprott, J.C. Simple chaotic flows with a line equilibrium. Chaos Solitons Fractals 2013, 57, 79-84. [CrossRef]

11. Pham, V.-T.; Jafari, S.; Volos, C.; Wang, X.; Golpayegani, S.M.R.H. Is that really hidden? The presence of complex fixed-points in chaotic flows with no equilibria. Int. J. Bifurc. Chaos 2014, 24, 1450146. [CrossRef]

12. Pham, V.-T.; Volos, C.K.; Jafari, S.; Wei, Z.; Wang, X. Constructing a novel no-equilibrium chaotic system. Int. J. Bifurc. Chaos 2014, 24, 1450073. [CrossRef]

13. Pham, V.-T.; Vaidyanathan, S.; Volos, C.K.; Jafari, S. Hidden attractors in a chaotic system with an exponential nonlinear term. Eur. Phys. J. Spec. Top. 2015, 224, 1507-1517. [CrossRef]

14. Tahir, F.R.; Pham, V.-T.; Volos, C.K.; Wang, X. A novel no-equilibrium chaotic system with multiwing butterfly attractors. Int. J. Bifurc. Chaos 2015, 25, 1550056. [CrossRef]

15. Molaie, M.; Jafari, S.; Sprott, J.C.; Golpayegani, S.M.R.H. Simple chaotic flows with one stable equilibrium. Int. J. Bifurc. Chaos 2013, 23, 1350188. [CrossRef]

16. Lao, S.-K.; Shekofteh, Y.; Jafari, S.; Sprott, J.C. Cost function based on Gaussian mixture model for parameter estimation of a chaotic circuit with a hidden attractor. Int. J. Bifurc. Chaos 2014, 24, 1450010. [CrossRef]

17. Shahzad, M.; Pham, V.-T.; Ahmad, M.A.; Jafari, S.; Hadaeghi, F. Synchronization and circuit design of a chaotic system with coexisting hidden attractors. Eur. Phys. J. Spec. Top. 2015, 224, 1637-1652. [CrossRef]

18. Kingni, S.T.; Jafari, S.; Simo, H.; Woafo, P. Three-dimensional chaotic autonomous system with only one stable equilibrium: Analysis, circuit design, parameter estimation, control, synchronization and its fractional-order form. Eur. Phys. J. 2014, 129, 76. [CrossRef]

19. Wei, Z. Dynamical behaviors of a chaotic system with no equilibria. Phys. Lett. A 2011, 376, 102-108. [CrossRef]

20. Leonov, G.A.; Kuznetsov, N.V.; Vagaitsev, V.I. Localization of hidden Chua's attractors. Phys. Lett. A 2011, 23, 2230-2233. [CrossRef] 
21. Bragin, V.O.; Vagaitsev, V.I.; Kuznetsov, N.V.; Leonov, G.A. Algorithms for finding hidden oscillations in nonlinear systems: The Aizerman and Kalman conjectures and Chua's circuits. J. Comput. Syst. Sci. Int. 2011, 50, 511-543. [CrossRef]

22. Leonov, G.A.; Vagaitsev, V.I.; Kuznetsov, N.V. Algorithm for localizing Chua attractors based on the harmonic linearization method. Dokl. Math. 2010, 82, 693-696. [CrossRef]

23. Kuznetsov, N.V.; Leonov, G.A.; Vagaitsev, V.I. Analytical-numerical method for attractor localization of generalized Chua's system. IFAC Proc. Vol. 2010, 4, 29-33. [CrossRef]

24. Sprott, J.C.; Jafari, S.; Pham, V.-T.; Hosseini, Z.S. A chaotic system with a single unstable node. Phys. Lett. A 2015, 379, 2030-2036. [CrossRef]

25. May, R.M. Simple mathematical models with very complicated dynamics. Nature 1976, $261,459-467$. [CrossRef] [PubMed]

26. Pierre, C.; Jean-Pierre, E. Iterated Map on the Interval as Dynamical Systems; Springer: Berlin, Germany, 1980.

27. Hénon, M. A two-dimensional mapping with a strange attractor. Commun. Math. Phys. 1976, 50, 69-77. [CrossRef]

28. Chen, G.; Mao, Y.; Chui, C.K. A symmetric image encryption scheme based on 3D chaotic cat maps. Chaos Soliton Fractals 2004, 21, 749-761. [CrossRef]

29. Jafari, S.; Pham, T.; Moghtadaei, M.; Kingni, S.T. The relationship between chaotic maps and some chaotic systems with hidden attractors. Int. J. Bifurc. Chaos 2016, 26, 1650211. [CrossRef]

30. Jiang, H.B.; Liu, Y.; Wei, Z.; Zhang, L.P. Hidden chaotic attractors in a class of two-dimensional maps. Nonlinear Dyn. 2016, 85, 2719-2727. [CrossRef]

31. Franks, J.M. Invariant sets of hyperbolic toral automorphisms. Am. J. Math. 1977, 99, 1089-1095. [CrossRef]

32. José, L.V.; Esteban, T.C.; Ángel, R.V. A switched-capacitor skew-tent map implementation for random number generation. Int. J. Circ. Theor. Appl. 2017, 45, 305-315.

33. Luis, G.F.; Esteban, T.P.; Esteban, T.C.; Cuauhtemoc, M.L. Hardware implementation of pseudo-random number generators based on chaotic maps. Nonlinear Dyn. 2017, 90, 1661-1670.

34. Ferrenberg, A.M.; Landau, D.P.; Wong, Y.J. Monte Carlo simulations: Hidden errors from "good" random number generators. Phys. Rev. Lett. 1992, 69, 3382-3384. [CrossRef] [PubMed]

35. Kyle, J.P.; Richard, J.P. Analyzing logistic map pseudorandom number generators for periodicity induced by finite precision floating-point representation. Chaos Solut. Fractals 2012, 43, 238-245.

36. Pincus, S.M. Approximate entropy as a measure of system complexity. Proc. Natl. Acad. Sci. USA 1991, 88, 2297-2301. [CrossRef] [PubMed]

37. Pincus, S. Approximate entropy (ApEn) as a complexity measure. Chaos Interdiscipl. J. Nonlinear Sci. 1995, 5, 110-117. [CrossRef] [PubMed]

38. Xu, G.H.; Shekofteh, Y.; Akgül, A.; Li, C.B.; Panahi, S. A New Chaotic System with a Self-Excited Attractor: Entropy Measurement, Signal Encryption, and Parameter Estimation. Entropy 2018, 20, 86. [CrossRef]

39. Koyuncu, İ; Özcerit, A.T. The design and realization of a new high speed FPGA-based chaotic true random number generator. Comput. Electr. Eng. 2016, 58, 203-214. [CrossRef]

(c) 2018 by the authors. Licensee MDPI, Basel, Switzerland. This article is an open access article distributed under the terms and conditions of the Creative Commons Attribution (CC BY) license (http:/ / creativecommons.org/licenses/by/4.0/). 